Supplement of Nat. Hazards Earth Syst. Sci., 20, 921-931, 2020

https://doi.org/10.5194/nhess-20-921-2020-supplement

(c) Author(s) 2020. This work is distributed under

the Creative Commons Attribution 4.0 License.

(c) (1)

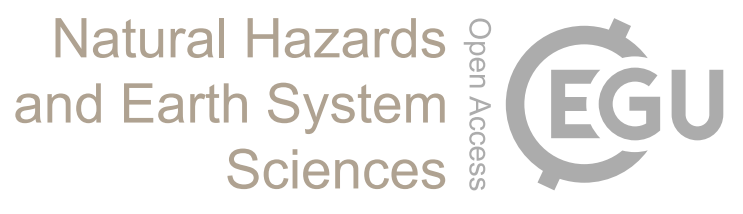

Supplement of

\title{
Design and implementation of a mobile device app for network-based earthquake early warning systems (EEWSs): application to the PRESTo EEWS in southern Italy
}

Simona Colombelli et al.

Correspondence to: Simona Colombelli (simona.colombelli@unina.it)

The copyright of individual parts of the supplement might differ from the CC BY 4.0 License. 


\section{Text S1: Definition of shaking duration and theoretical background}

The theoretical formulation for the shaking duration computation grounds on the original definition of event duration, as proposed by Real and Teng (1973), who measured the duration of an earthquake based on the attenuation time of the coda waves. The only difference is related to the threshold level for the declaration of the end, that was a value comparable to the pre-event level of noise, in the case of event duration, while here we set a threshold value of $0.2 \mathrm{~cm} / \mathrm{s}$, to identify the end of the strongest shaking.

The shaking duration indeed is defined at each station as the time interval between the arrival of the P-wave and the moment at which the ground velocity decreases down to a predefined threshold value $(0.2 \mathrm{~cm} / \mathrm{s})$. The shaking duration is measured on the horizontal components of the ground velocity and Figure S1 shows an example of shaking duration measurement along the record. Specifically, for each available station, the algorithm waits for the P-wave arrival (measured on the vertical component, red line)) and then continuously monitor the recorded ground shaking on the horizontal components. The absolute peak velocity (PGV) is first identified (blue line) and then the algorithm looks for the end time (green line) as the moment at which the recorded velocity decreases down to the threshold level (horizontal dashed line).

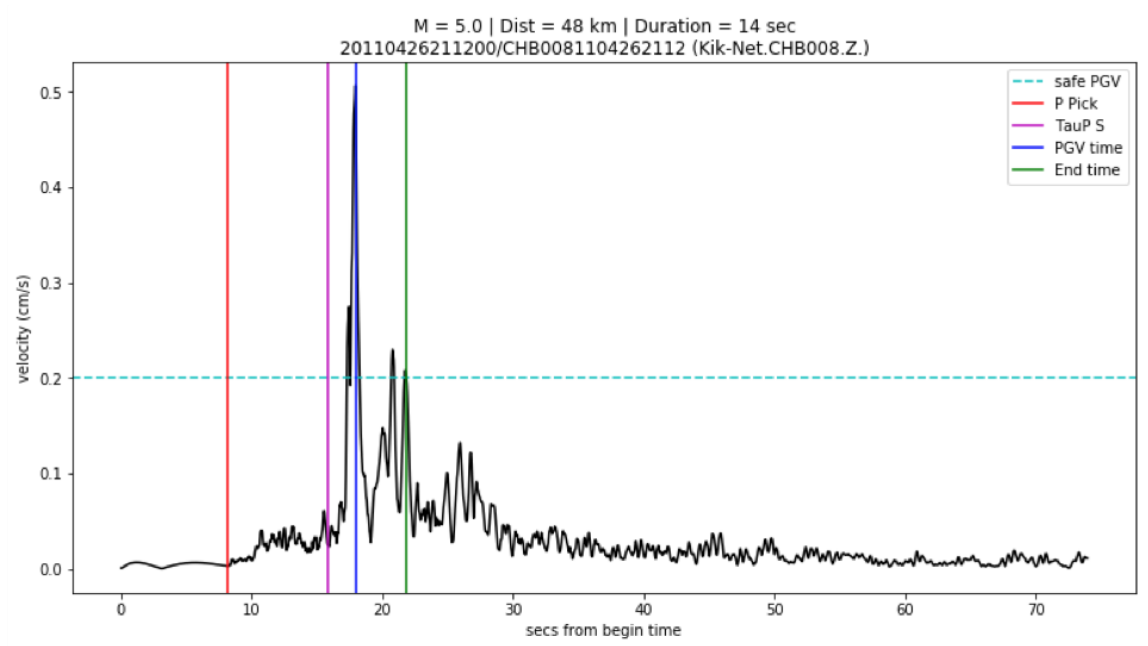

Figure S1: definition of shaking duration. The figure shows an example of shaking duration measurement. The shaking duration is measured as the difference between the arrival of the P-wave (red line) and the moment at which the shaking goes down to the threshold level of $0.2 \mathrm{~cm} / \mathrm{s}$ (green line).

Following the theoretical formulation of Real and Teng (1973), the duration of an earthquake $(\Delta)$ is related to the event magnitude $\mathrm{M}$ and to the distance $\mathrm{R}$, through a log-linear relationship of the type:

$$
\log (\Delta)=\alpha+\beta \mathrm{M}+\gamma \mathrm{R}
$$

where $\alpha, \beta$ and $\gamma$ are empirically derived coefficients. Several authors (REF) have shown that for regional distances, smaller than 100-150 km, the dependency on distance (expressed through the $\gamma$ term), can be negligible, with the main contribution to the earthquake duration provided by the event magnitude. Being the shaking duration conceptually similar to the duration of the event, we adopted the original formulation for the calibration of the following empirical scaling lows, as described hereinafter. 


\section{Text S2: Dataset description}

We collected a set of earthquake records from Italian and Japanese earthquakes, in the magnitude range between 3.5 and 9, and in the distance range from 0 to $200 \mathrm{~km}$, which is the regional distance range the APP is targeted to, for a total of 4036, 3-component records. For each available record, we measure the shaking duration on the horizontal components of the ground velocity, as the time interval between the arrival of the P-wave and the moment at which the ground velocity decreases down to a predefined threshold value $(0.2 \mathrm{~cm} / \mathrm{s})$. We then preliminarily computed the average duration for different classes of magnitude and distances (magnitude bins equal to 0.5 , distance bins of $10 \mathrm{~km}$ ) and looked at the scaling of these durations with both parameters. Figure S2 shows the average durations as a function of distance, with a color representing the magnitude.

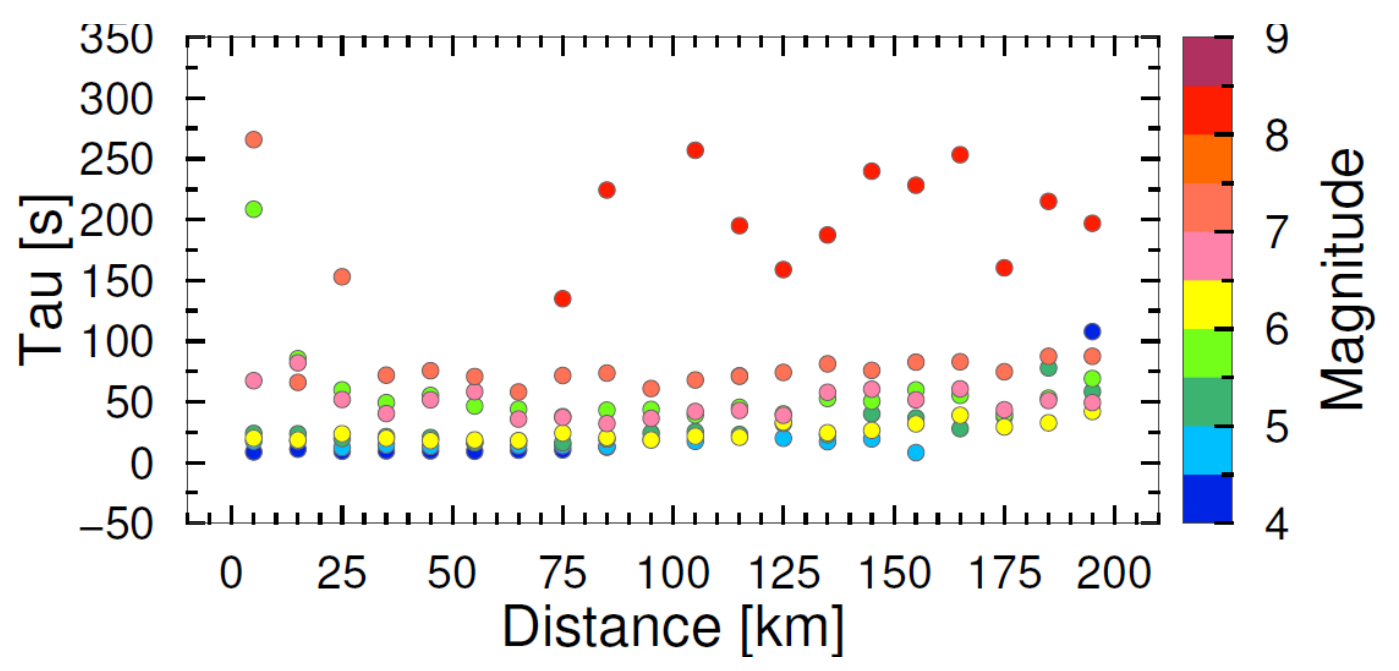

Figure S2: average shaking duration vs. distance and magnitude. The figure shows the average shaking duration as a function of distance, and with a color representing the earthquake magnitude. The average durations are computed in in magnitude and distance bin (respectively, 0.5 and $10 \mathrm{~km}$ ).

As expected from the theory and as it is evident from the plot of Figure S2, the shaking duration is pretty insensitive to the distance, while it mainly scales with the event magnitude, with short duration values ( $\tau_{\text {shake }}<10-20$ s) associated to small magnitudes (M 4-5) and longer durations ( $\tau_{\text {shake }} \approx 50$ s) for the moderate events (M6-7) and very long duration values ( $\tau_{\text {shake }}>150-200$ s) during the occurrence of giant earthquakes (M9).

Once the dependency on distance is excluded, the shaking duration is finally related to the earthquake magnitude. To this purpose, for each magnitude bin (of 0.5 ), we computed the average shaking duration with its uncertainty. Then, through a weighted fitting procedure, we empirically estimated the coefficients of the log-linear correlation of the type:

$$
\log \left(\tau_{\text {shake }}\right)=\mathrm{a}+\mathrm{bM}
$$

where $\mathrm{M}$ is the magnitude (central value of the bin) $\mathrm{a}$ and $\mathrm{b}$ are the estimated coefficients. The plot of Figure 7 in the main text shows the scaling between duration and magnitude, and the estimated coefficients. 